\title{
RAPID EVOLUTION OF GALACTIC NUCLEI
}

\author{
WILLIAM G. TIFFT
}

University of Arizona, Tucson, Ariz., U.S.A.

\begin{abstract}
An evolutionary sequence is suggested for Virgo cluster galaxies, in a radial velocity-nuclear magnitude diagram. This evolution, on a time scale of about $10^{8} \mathrm{yr}$, is discussed in terms of the morphology and radial color variations of the galaxies, nuclear size and radio emission. An area scan and profiles of nuclei, obtained with image dissector equipment, are presented.
\end{abstract}

Four-color five-aperture photometry along with visual nuclear sizes and descriptions of all bright Virgo Cluster galaxies has been published (Tifft, 1969). Apertures ranged from 64 to 10 arc sec. On the basis of absolute colors and radial color variations, as shown in Figure 1, both elliptical (E) and spiral (S) galaxies were divided into four

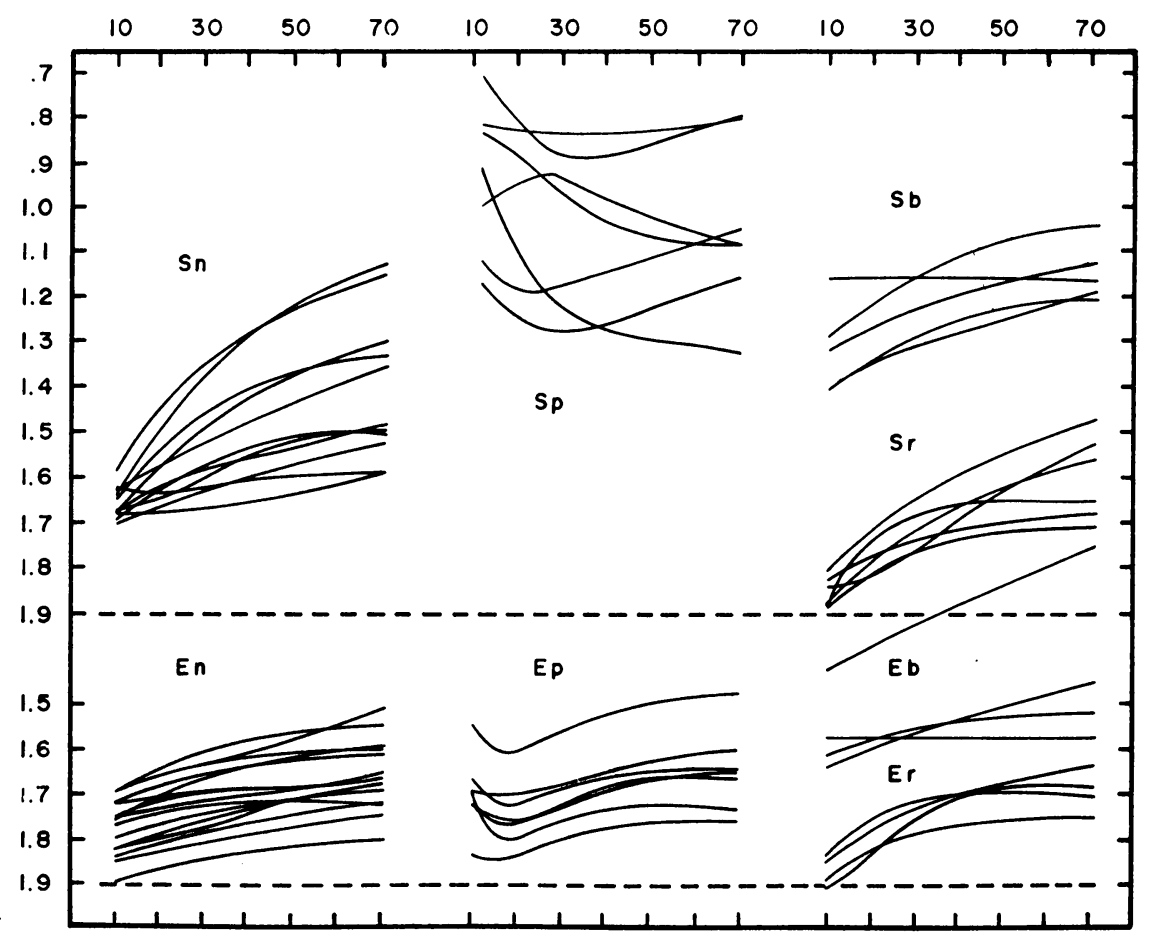

Fig. 1. Color Index (1-3) as a function of aperture diameter in arc sec for the eight basic photometric types of galaxies.

color classes; normal $(n)$, blue nucleated $(p)$, red nucleated $(r)$, and systematically blue $(b)$.

Comparison of photometric type with red-shift and with nuclear light concentration indicated correlations in both cases. The $r$ nuclei show sharp bright nuclei and are 
present in galaxies including many of the most extreme velocities. The $p$ nuclei tend to be diffuse. Because of these correlations a comparison of velocity directly with light concentration was done using the visual estimates of nuclear size. Figure 2 shows the correlation for galaxies in the $\mathrm{E}$ and $\mathrm{S}$ subsystems of the Virgo Cluster.

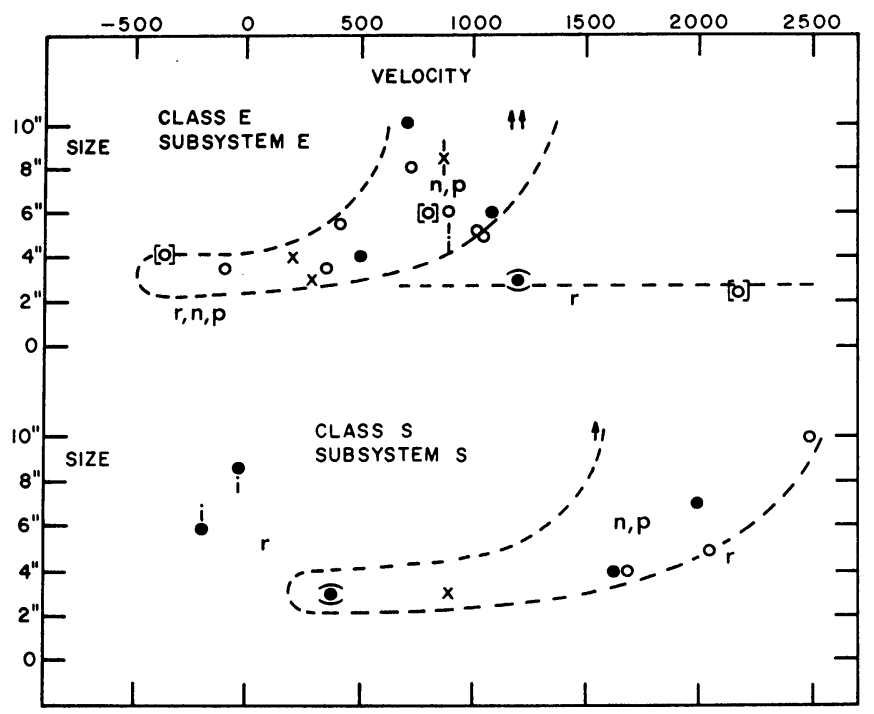

Fig. 2. The correlation of velocity with apparent nuclear region dimensions in arc sec for galaxies in the $\mathrm{E}$ and $\mathrm{S}$ subsystems of the Virgo Cluster. All galaxies $m_{H}<12.0$ plus two fainter. i Highly inclined, size indeterminate; $\odot$ Called stellar, 3 " size assigned ; $\bigcirc$ No radio data; $\bigcirc$ Radio data negative; $x$ Small radio source; | Large radio source; [] Radio source nearby.

Velocity and size are correlated with higher velocity corresponding to less stellar-like nuclei. There are several possible interpretations.

(1) The galaxies could be redistributed into subgroups at different distances as has been suggested for the $\mathrm{E}$ and $\mathrm{S}$ subgroups. This would, however, imply that nearby dwarfs have sharp bright nuclei.

(2) Mass radial motion of stars could be invoked at some stage in a transient evolutionary phase of galaxy development.

(3) Gravitational or some other form of non-Doppler redshift might be invoked.

To discriminate between possibilities, the correlation of nuclear magnitude with velocity was considered. Magnitudes at $10 \mathrm{arc} \mathrm{sec}$ where the nuclei dominate were plotted against velocity as shown in Figure 3. Elliptical galaxies occupy two welldefined linear regions denoted as the $r p$ and $n$ branches. The total vertical spread, including the Virgo X subsystem, is no more than one would expect from the natural depth of a spherical cluster. Spiral galaxies divide into two types. One group parallels the E pattern slightly displaced; most spirals occupy a fainter zone denoted the $\mathrm{S}$ region.

The grouping in Figure 3 is not the same as in Figure 2. There seems to be no way the galaxies can be grouped to preserve the concept of several simple clusters with 


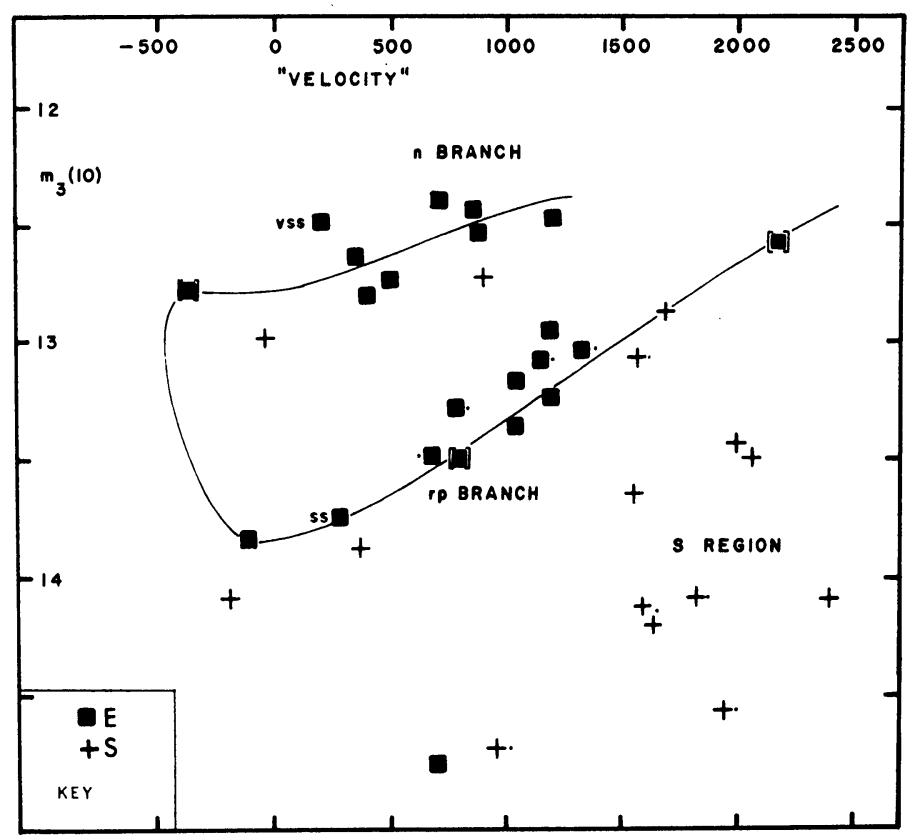

Fig. 3. 'Velocity' as a function of band 3 magnitude for galaxy nuclei isolated in a 10 arc sec aperture. Symbols with a small dot beside indicate galaxies in the $\mathrm{X}$ subsystem of the Virgo Cluster. Otherwise, galaxies belong to the $\mathrm{E}$ or $\mathrm{S}$ subsystems. All galaxies with Shapley-Ames magnitude $<12$ are shown plus two fainter ones. Information on small radio sources is included in the figure. vss $=$ unresolved small radio source (NGC 4552); ss = resolved small radio source (NGC 4550); [] about a symbol means a radio source is nearby but not at the galaxy.

random internal velocity dispersion. Instead, Figure 3 is interpreted to show an empirical evolutionary pattern for galaxy nuclei in one cluster. There seems no way that the velocity can be interpreted as a Doppler shift of the galaxy mass centers, although it might in part arise from mass radial motion about the nuclei. Such motion may play a role in the low velocity end of the diagram and will be considered later. However, one cannot sustain for times in excess of a million years the great correlated implosions and explosions implied at the extreme differential velocities, and one is led to the conclusion that the differential velocities at the extreme positive end of the diagram at least are probably not real Doppler shifts at all. The cluster as a whole will still have an associated group Doppler velocity even if the differential velocities have another explanation. The wide velocity spread along the tracks leaves the group velocity rather indeterminate, however. The notation $V$ will be used subsequently unless real mass center velocity is implied.

A number of galaxies in the Virgo region have been called foreground objects, most often because of low or negative $V$. Holmberg (1961) pointed out that the negative $V$ galaxies are larger extended objects which might imply nearness. An alternative hypothesis is that these galaxies are in an expanding core-halo phase of evolution which could account for the extended halos and the negative $V$ which might 
be a true Doppler velocity in this case. Burbidge and Demoulin (1969) have studied an interesting negative $V$ dwarf galaxy in the Virgo region and concluded that rapid radial motion of surrounding material was possible.

The three galaxies with extended radio sources, NGC 4486, 4472, and 4374, are grouped at the tip of the $n$ branch. This is best seen in Figure 4 where larger aperture photometry is shown. Twenty-six arc sec photometry minimizes effects of nuclear

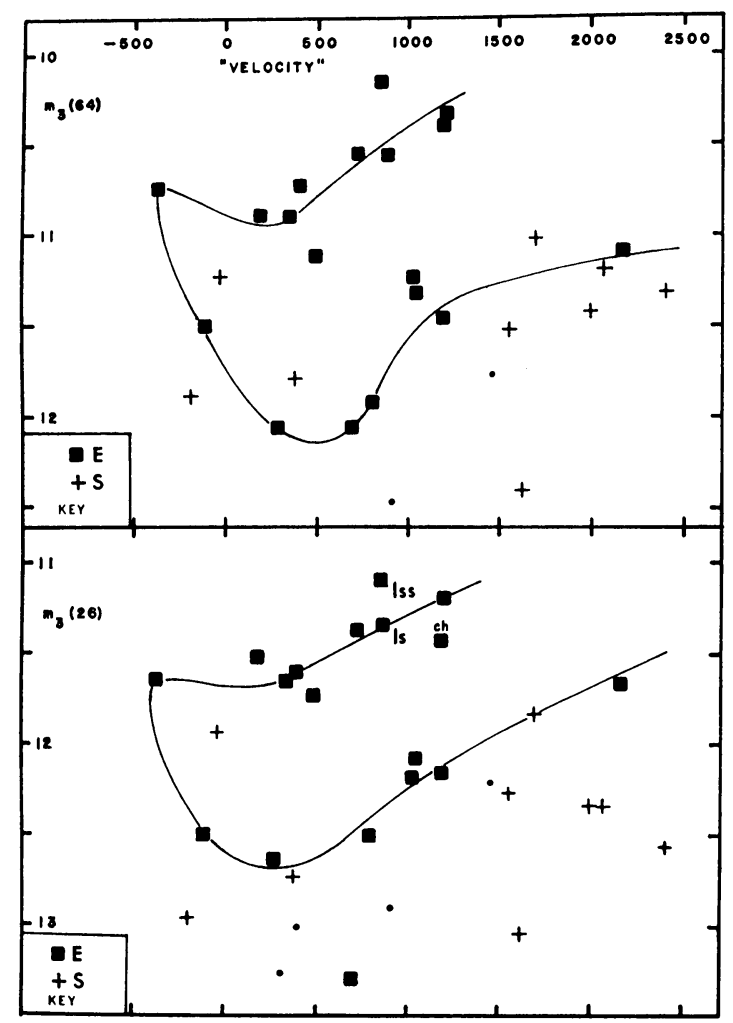

Fig. 4. 'Velocity' as a function of band 3 magnitude for galaxy nuclei isolated in 64 and 26 arc sec apertures. Only galaxies in the E or S subsystems of the Virgo Cluster are shown. All galaxies with Shapley-Ames magnitude $<12$ are shown plus two fainter ones. Information on large radio sources is included in the figure. 1s = large radio source (NGC 4374); lss = large and resolved small radio source (NGC 4472); ch = complex core-halo radio source (NGC 4486). Small dots indicate faint E galaxies which have partial magnitude data available. At smaller apertures they appear to converge toward the main branch of the diagram.

sharpness or diffuseness which is strongly correlated with photometry type on the $n$ branch. NGC 4486 is believed to be an extremely massive object, consequently it would be surprising to find it anywhere but near the tip of the evolutionary track. A good correlation exists between small radio sources and galaxy photometry type (Tifft, 1970) as shown in Figure 5. The two small sources in the Virgo Cluster lie near the low $V$ end of the $n$ and $r p$ branches, one on each branch. 


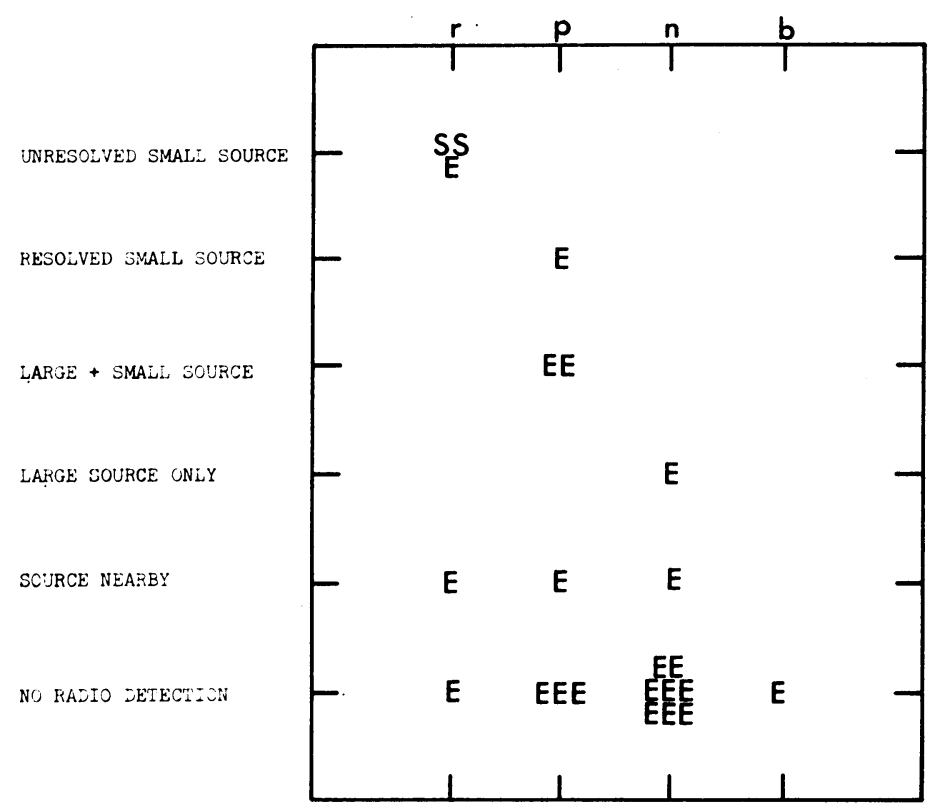

Fig. 5. The correlation of radio source type with nuclear color class for $\mathrm{E}$ and $\mathrm{S}$ galaxies. All objects with both radio data (Heeschen or Wade) and nuclear color classification are shown.

Figure 4 contains information on a few additional galaxies and on the behavior of the envelope surrounding the 10 arc sec nuclear region. Scatter increases markedly as more and more non-nuclear information is included in the photometry. However, the pattern from Figure 3 remains visible. Ten arc sec photometry is not available for faint galaxies in the Virgo region, however, a few available larger aperture measures are shown in Figure 4. They appear to converge toward the $r p$ branch as one shifts to smaller aperture. Realistically, one might expect a series of tracks displaced from the main one illustrated in accord with fundamental parameters such as angular momentum and total system mass. Numerous dwarf galaxies are known in the Virgo Cluster covering a wide $V$ range including negative values. It is not likely that the dwarf systems will correspond directly with the branch defined by the luminous objects, hence, the $r p$ branch is more likely an upper envelope. On the basis of presently available photometry, however, there is no evidence that the upper envelope structure in the figures will be radically changed.

The relatively uniform density of points along the tracks in Figure 3 implies a relatively uniform rate of evolution, somewhat faster at low $V$. The $r p$ branch is presumably incomplete because of the lower magnitude cut-off in the available photometry, consequently, the frequency of points on the $r p$ branch cannot be assessed in detail. The $n$ branch must be nearly complete.

It was earlier suggested that the negative $V$ portion of the track represents an evolutionary step with rapid halo development. Whatever the details in the stage it appears to be a rapid event. Photographs of NGC 4438 show two long filaments 
which are only slightly wound. The implication is that outflow is rapid and the stage occupies less than a galaxy rotation. Thus, the transition from the $r p$ to $n$ branches may require only a few times $10^{8} \mathrm{yr}$ and possibly less if a large part of the differential $V$ is a true mass motion velocity. Since galaxies are seen in this stage, and objects are not that much more concentrated on the $n$ branch, one is led to hypothesize that the evolution along the upper portions and possibly the entire track may occur in less than $10^{9} \mathrm{yr}$.

Nuclear size appears to be a minimum just preceding the transition from the $r p$ to $n$ branches and again is very small at the start of the $n$ branch. Proceeding down the $n$ branch the nuclear sizes become smoothly and rapidly larger. The two small radio sources appear to occur near the points of minimum nuclear size. A sudden loss of appreciable nuclear region mass by ejection or gravitational radiation at the end of the $r p$ branch could initiate the halo expansion. One rebound in the mass motion near the nucleus appears to occur at the end of the halo phase and then the nuclear region expands diffusely. Large numbers of outer stars may be lost completely by the galaxy which might thus form the vast outer halo of stars as seen about M87. Rotation of the axes of outer luminosity profiles would also occur as observed in many galaxies. Velocity dispersion appears to grow smoothly along the $n$ branch, as it should if less and less phase correlation remains from the original stellar motions as the disturbed galaxy seeks a new equilibrium condition. The velocity dispersion will bear little relation to system mass.

For further evidence of rapid evolution, one might consider the complex ring and other unstable structures seen in many galaxies and ask how these could be maintained long enough to be seen if a normal quiescent state were the rule. Finally, there is no fundamental reason why galaxy evolution can not be rapid. The evolution of stars is determined by the core and the envelope follows as directed. Knowing as little as we do about galaxy nuclei, we cannot say that they do not play an analogous role in driving the galactic envelope where a steady state need not be the rule. The observations of large amounts of gravitational radiation from the nucleus of our Galaxy could be consistent with rapid evolution of nuclei and would result in mass motion in the envelope. Unusual features seen in rotation curves of various galaxies may point in the same direction.

Adopting the hypothesis of rapid evolution, one may ask where it leads. The placing of the Virgo Cluster galaxies on an evolutionary pattern where a large part of the differential $V$ does not represent mass center motion can completely eliminate the Virial Theorem mass discrepancy for the cluster. Stability for the Virgo Cluster leads to an examination of other apparently unstable galaxy configurations, including extreme cases such as Stephan's Quintet, the IC 3481 group, and the VV 172 chain. Assuming the $V-m$ evolutionary track continues to higher $V$, the position occupied by any group is determined by its time of origin less the light travel time. Either very distant groups or groups of recent origin could map onto higher $V$ segments of the track. This is suggested to be the case for the supposedly unstable galaxy configurations. The existence of a non-Doppler differential $V$ effect in galaxies could also 
explain the mass discrepancies derived from double galaxies and large clusters. Discordance in $V$ within clusters, groups, or pairs will not imply gravitational instability.

The observed red shift of any galaxy can be represented as the sum of its group Hubble velocity and the differential $V$. The existence of one low $V$ value in Stephan's Quintet and the IC 3481 group is significant since it suggests that these groups might be evolving toward a low $V$ point which could imply a small Hubble parameter. However, systematic velocities of major clusters make this unlikely. If the rapid rates of evolution implied in this paper are real, very distant clusters might begin to show
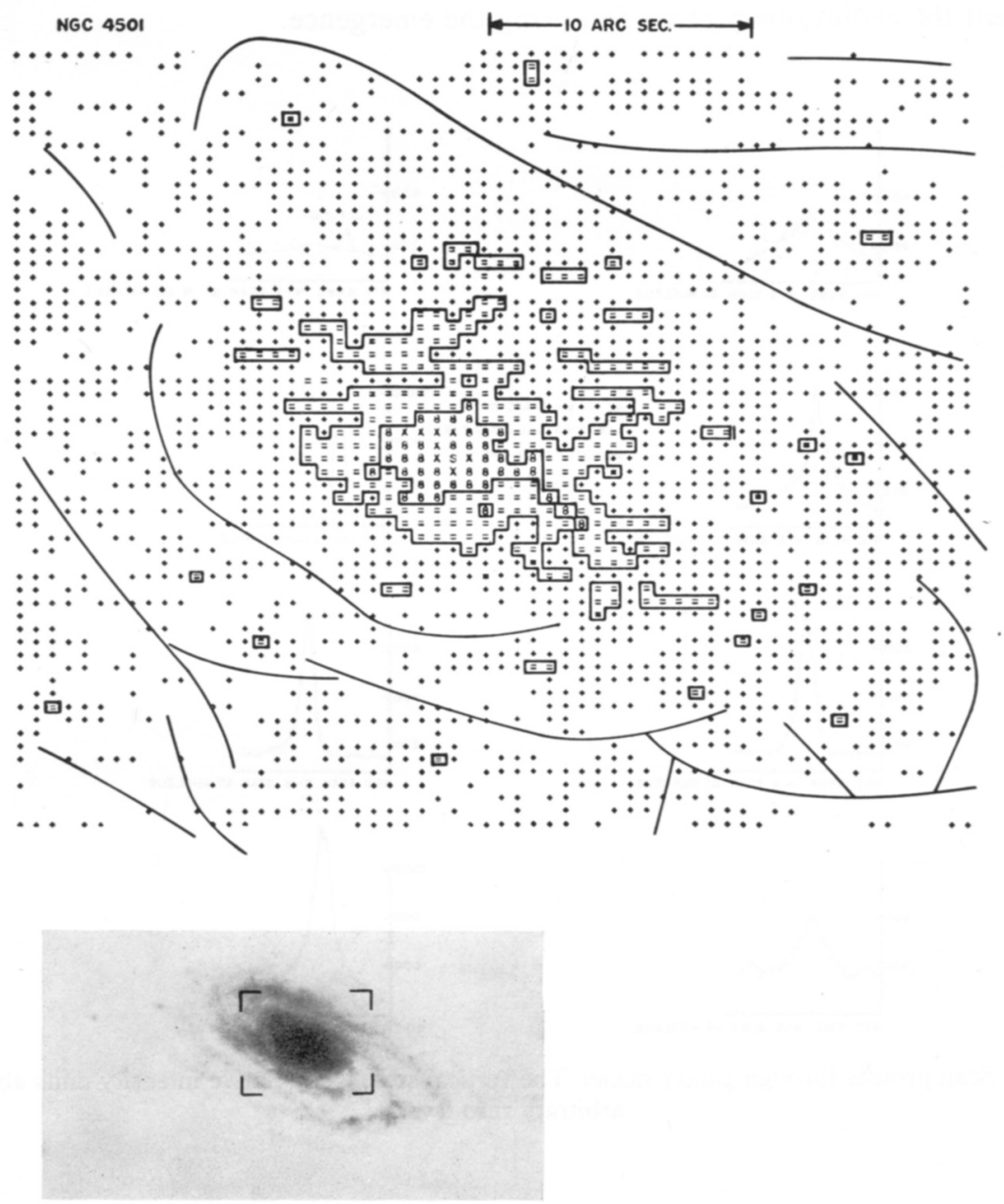

Fig. 6. Computer processed picture of a $60 \times 60$ area scan of the nuclear region of NGC 4501 . Dark lanes have been indicated by light lines and two isophotes have been drawn in for clarity. The scan limits are indicated on a small scale direct photograph included for couparison. Scan scale is $0.6 \mathrm{arc} \mathrm{sec}$ between points for total scan width of $38 \mathrm{arc} \mathrm{sec}$. The scanning aperture has an effective diameter of 1.5 arc sec. 
sensible differences in total galaxy luminosity or size which could severely complicate determination of $q$ regardless of the value of $H$.

Continued extension of the $r p$ branch in the $V-m$ diagram could conceivably lead to the QSS which could be early stages of galaxies or clusters of galaxies, or could represent stages in galaxy evolution. Non-uniform distributions in $z$ could be produced either by variation of the rate of evolution along the track or the total luminosity of sections of the track. The absence or near absence of QSS objects in clusters would be expected if clusters occupy 'local' portions of the track and QSS objects lie at remote points. The 'expanding core' hypothesis which has been most recently discussed by Harrison (1970) offers an interesting means of origin of the $r p$ branch which would represent the evolutionary chain following the emergence.
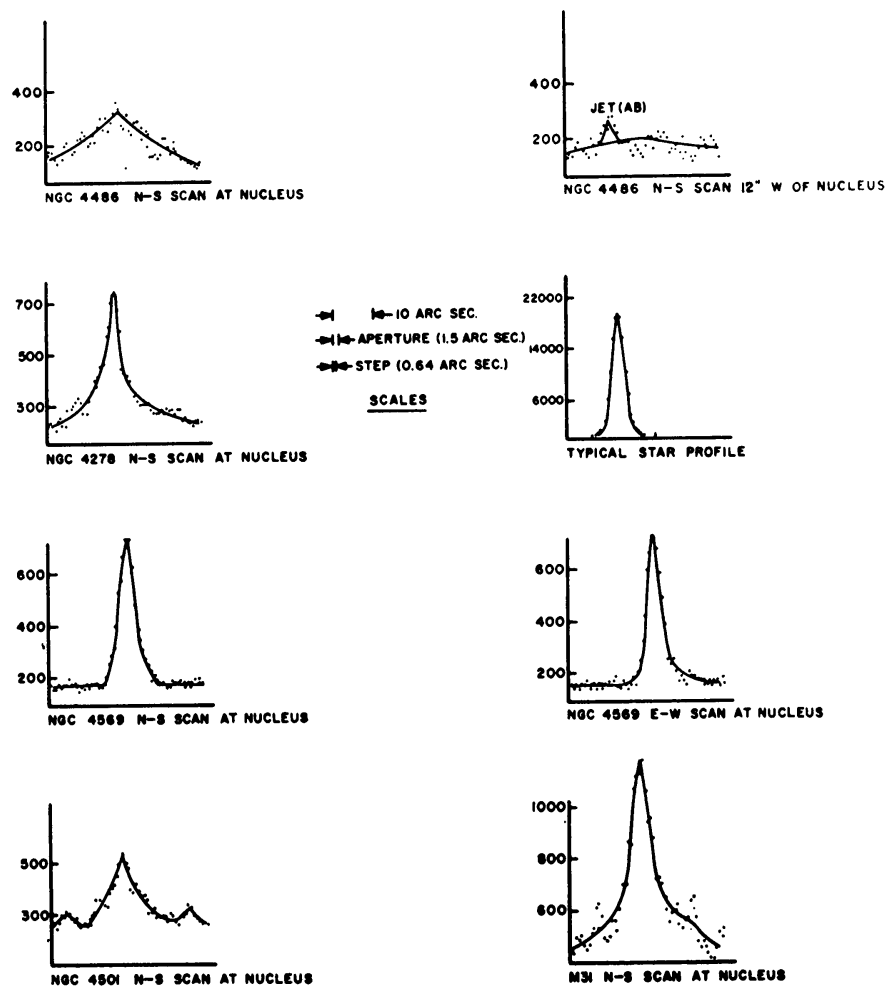

Fig. 7. Scan profiles through galaxy nuclei. The vertical scale is in relative intensity units above an arbitrary zero level.

Many aspect of the hypothesis of rapidly evolving galaxies are available for observational and theoretical development. Refined data on photometry of galaxy nuclei will be important. Figure 6 illustrates a preliminary area scan of the nuclear region of NGC 4501 obtained with image dissector equipment developed under NASA 
Grant NGR 03-002-032. Figure 7 shows a set of typical profiles through or near galaxy nuclei obtained with the same equipment which will be used to map color and brightness distributions in galaxy nuclei.

\section{References}

Burbidge, E. M. and Demoulin, M. H.: 1969, Astrophys. J. Letters 157, L155.

Harrison, E. R.: 1970, Monthly Notices Roy. Astron. Soc. 148, 119.

Holmberg, E.: 1961, Astron. J. 66, 620.

Tifft, W. G.: 1969, Astron. J. 74, 354.

Tifft, W. G.: 1970, Astrophys. Letters, 7, 7. 